\title{
Implementation of Neighbor Discovery Protocol for Underwater Acoustic Communications
}

\author{
Manjula R. Bharamagoudra*, SunilKumar S. Manvi \\ Wireless Information System Research Lab, Department of Electronics and Communication Engineering, \\ REVA Institute of Technology and Management, REVA University, Bengaluru, Karnataka, India. \\ * Corresponding author. Email: manjularb@rediffmail.com \\ Manuscript submitted March 25, 2015; accepted August 18, 2015. \\ doi: 10.17706/ijcee.2015.7.5.325-334
}

\begin{abstract}
Underwater Acoustic Sensor Networks (UASN) consisting of sensor nodes and underwater gateways (routers) have wide variety of applications, which include environmental monitoring, scientific exploration, surveillance etc. Ad hoc nature of these networks in terms of mobility due to water current pose challenges in topology management. Neighbor discovery is an important issue in topology management which is required to auto configure the node addresses, and establish communication links among the nodes to perform routing management. The media access control (MAC) protocol may utilise the neighbor discovery information to decide the accessibility of the media by nodes so as to reduce the delays and increase throughput. Thus, there is a necessity of integrating MAC protocol and neighbor discovery mechanism in UASN. In this paper, we describe the architectural elements of neighbor discovery protocol module implementation in NS3. The implementation models surface and underwater interactions, mobility, and channel conditions required for network level simulation. The module implements two main classes namely, NeighborDiscoveryProtocol and NeighborDiscoveryCache, which enable the protocol interactions. We present the preliminary results from implemented module using NS3.
\end{abstract}

Key words: Direction of arrival estimation, neighbor discovery protocol implementation, underwater acoustic networks.

\section{Introduction}

Around two-third surface of earth covered by oceans has received considerable interest in the field of underwater acoustic networks from the research community for its potential information. The Ad hoc Underwater Acoustic Sensor Networks (UWASNs) consists of a variable number of sensors and vehicles that are deployed to perform collaborative monitoring tasks over a given area [1]. The potential applications are in field of monitoring oceanic environments for scientific, security, military needs and monitoring the strong influences and impact of climate regulation such as temperature, salinity and wave pressures, nutrient production, oil retrieval and transportation. The development of low cost, low size, light weight sensor nodes and the improvement in the methods of deployment of these devices physically near the objects of interest brings new opportunities for the researchers to collect and analyse the data for human benefit. The environment of underwater poses many challenges in terms of the bandwidth, node mobility, neighbor discovery, error rate, propagation delay, memory, battery power and so on [2]. Due to the water currents, the sensor nodes and vehicles (mobile nodes) drift from the positions of deployment in a random fashion and hence the topology changes. The task of establishing the communication link in changing topology is vital as 
the network operation begins only after each node has discovered its neighbors and established the paths to the other nodes even when the nodes have no knowledge of their neighbor's IDs or locations. The objective of Neighbor discovery protocol is to inform the neighbor's identity and build the routing tables that can be further utilized by the network layer to establish the communication path.

Neighbor discovery is the process by which a node in a network determines the total number and identity of other nodes in its vicinity. It is a basic building block of many protocols including localization, routing, and cluster management. Also network layer algorithm and many media access control mechanisms rely on accurate neighbor information. Neighbor discovery is especially important for proper functioning of wireless networks.

The proposed neighbor discovery uses the directional sectored antennas for the transmission and reception of the frames. The best estimate of received signals is computed using the various Direction of Arrival (DOA) algorithms [3] for discovery. The advantage of using the DOA estimation is that the neighbors linked with high link quality are estimated and thus network throughput will be improved. Directional antennas have a number of advantages [4] over omni-directional antennas for ad hoc networking. By focusing energy only in the intended direction, directional antennas significantly increase the potential for spatial reuse. They provide a longer range and/or more stable links due to increased signal strength and reduced multipath components. Increased spatial reuse and longer ranges translate into higher ad hoc network capacity (more simultaneous transmissions and fewer hops), and longer ranges also provide richer connectivity. Further, since the spatial signature of the energy is reduced to a smaller area, chances of eavesdropping are reduced, and with "smart" antennas, steering of nulls allows suppression of unnecessary interference (such as jammers). However, unlike sensors with omnidirectional antenna sensors with directional antenna take longer time to discover their neighbors [5]. This is because although sensors may be within transmission range, the sender/receiver sensor may not necessarily be located within the given sector determined by the beaming antenna of the transmitting sensor.

The solutions for underwater neighbor discovery have been proposed based on the variants of successful terrestrial approaches. To the best of our knowledge, there is no MAC layer protocol for supporting the usage of directional antennas in ad hoc underwater networks. The issues to be addressed are exploitation of spatial diversity gains due to the use of directional antenna and in the presence of mobility, the MAC protocol [6] has to incorporate mechanisms by which a node can efficiently discover and track its neighbors.

The focus of our present work is on design and implementation of architectural elements of neighbor discovery protocol module based on direction of arrival estimation. The module implements two main classes namely NeighborDiscoveryProtocol and NeighborDiscoveryCache. The developed module is compliant with NS3 module standards and the module functionality comprises of two parts namely: NDP functionality and Signal Processing functions for DOA Estimation. The neighbor discovery is carried out using the direction of arrival estimation (DOA) algorithm [7].

The rest of paper has been organized as follows: Section 2 presents a review of the existing works. Section 3 presents the design architecture and Section 4 discusses the protocol operations. The simulation results and discussions are provided in Section 5. Section 6 concludes by summarizing our contributions and identifying directions for future research.

\section{Related Works}

Neighbor discovery has been extensively investigated for radio networks, but work in the field of underwater acoustic networks is extremely scarce. In this section, we briefly review the work related to neighbor discovery protocols. Efficient neighbor discovery algorithms in a synchronous network of sensors employing directional antenna with identical transmission/reception beam widths is discussed in [5]. The 
authors provide deterministic and randomized algorithms to accomplish neighbor discovery. A decentralized node discovery procedure for an underwater acoustic network is proposed in [8] in which the nodes use random access and transmit at the minimum power level required to reach a particular neighbor. If the neighbors are not reachable with the present power level, the power level will be increased and the process is repeated for node discovery. Power control is implemented in discrete levels and accounts for both the distance-dependent transmission loss and the channel fading. The authors assume that the nodes are deployed over a possibly large area that cannot be spanned in a single hop within the constraints of a finite power budget. The nodes operate in a distributed manner, that is, without a central station of the channel access and a random access environment. The goal is to design a protocol that is efficient in terms of energy consumption as well as the time it takes to complete.

A specific neighbor discovery procedure based on polling by a master node in a centralized configuration for an underwater acoustic network is discussed in [9]. The channel access was regulated through code division-multiple access. A similar kind of polling based neighbor discovery is discussed in [10]. The secured neighbor discovery protocol [11], discovers the secured neighbors considering the wormhole attacks in the network.

The exhaustive works have been carried out for node discovery in the terrestrial sensor networks. The work in [6] proposes a Polling based MAC protocol (PMAC) for mobile ad hoc networks. The PMAC exclusively uses directional antennas for transmission and reception of all the frames, the protocol facilitates the discovery of one-hop neighbors. Polling is also used to schedule the transmissions and receptions of information. The transmitter and receiver nodes point their antenna beams towards each other and carry on the communication exclusively in directional mode. A cross-layer approach to integrate neighbor discovery with the MAC protocol stems from the close interaction and dependence between the two functionalities. The authors discuss the inability of exclusively using directional antennas for both the transmission and reception of all MAC layer frames (control or data). A few of the works have been discussed as follows. The usage of directional antenna for the improvement of MAC layer performance is discussed in [12]. A reliable neighbor discovery protocol for mobile adhoc networks is presented in [13], and the work in [14] presents a secure neighbor discovery protocol for a compromised node in a static network. The Mobile Secure Neighbor Discovery (MSND) protocol which offers a measure of protection against wormholes by allowing participating mobile nodes to securely determine the neighbors is discussed in [15]. The work in [16] presents neighbor discovery algorithms for terrestrial ad hoc networks. The methodology adapted is to compute optimal parameter settings for neighbor discovery by considering clique topologies. It assumes that a node has knowledge of number of its one hop neighbors which is impractical for mobile network; the work does not consider neighbor maintenance. A probabilistic protocol for node discovery is discussed in [17], and family of probabilistic protocols, i.e., Birthday protocols for static ad hoc networks attempt to conserve energy during deployment phase while increasing the probability of neighbor discovery during the discovery phase [18].

\section{Design Architecture}

This section outlines the architectural elements of the Direction of Arrival Based Neighbor Discovery Protocol (DOA-NDP) implementation. The implementation models surface and underwater interactions, mobility, and channel conditions required for network level simulation. The module implements two main classes namely NS3::NeighborDiscoveryProtocol, NS3::NeighborDiscoveryCache which enables the protocol interactions. The module provides support for three propagation models namely, the Ideal channel model, the Thorp propagation model and the Bellhop propagation model. Each of these three propagation models provides power delay profile (PDP) contexts and utilizes a tapped delay line model to return the Propagation 
loss. The power delay profile information and the returned path loss is used to find the received signal strength and the corresponding intersymbol interference (ISI) information.

Fig. 1 shows the design architecture of the NS3 module developed and the class hierarchy of the Neighbor Discovery Protocol module for Underwater Communication. The developed module is compliant with NS3 module standards and derives from some kind of functionality provided by NS3::core, NS3::network and NS3::internet modules. The module functionality comprises of two parts namely: NDP functionality and Signal Processing functions for direction of arrival estimation. The NDP functionality contains classes for NDP::Protocol message handling, unreachability detection, packetization, and an NDP::CacheManager. On the other hand, the Signal Processing Module contains functions for supporting various computational operations required by DOA algorithms. The advantage of DOA estimation is that it finds the best estimate among multiple signals received in the presence of noise. The two components combined together form the DOA-NDP implementation, which utilizes the flexibility provided by DOA techniques to improve the efficiency of the Neighbor Discovery process at the MAC layer.

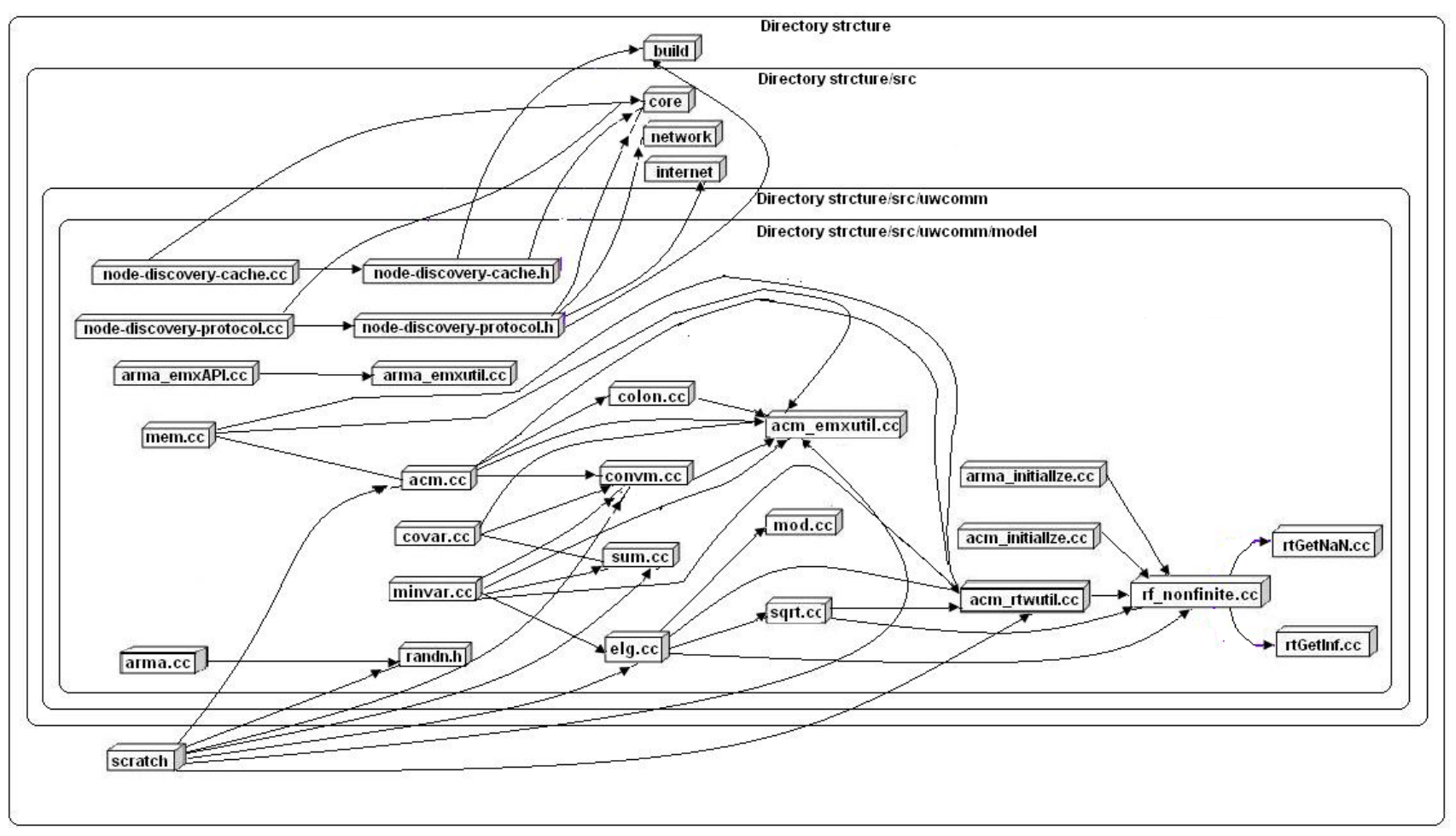

Fig. 1. Design architecture.

\subsection{Physical Layer Design}

The physical layer is responsible for packet handling and acquisition, error recovery, and for successful forwarding of packets to the MAC layer. The physical layer implements two models, the signal to noise ratio (SINR) model and the packet error rate (PER) model. These two models combined together are helpful in the successful determination of packet receptions. The above two models allocate a unique identification number for each packet received from the MAC layer. This identification number is used to calculate the error probabilities and the SINR information in the physical layer. Frequency Hopping (FH) FSK with a rate 1/2 convolutional coding scheme of constraint length 9 is implemented as the modulation and coding scheme. Error recovery is made possible through the use of a cyclic redundancy check (CRC) system capable of correcting up to 1 bit error. The DOA estimation is carried out using the various methods such as minimum variance, maximum entropy method, autoregressive and autoregressive moving average method. Based on 
the context of environment such as static or mobile different methods are suitable for finding the best estimate of the signal.

\subsection{MAC Layer Design}

The MAC layer design includes carrier sense mechanism. When the channel is busy (during carrier sensing), the nodes backoff by a random slot time as determined randomly (using a uniform distribution) and choose a slot to transmit information. The slot time durations can be configured during the simulation run via an attribute.

\section{Protocol Operations}

In this section, we will discuss the sequence of operations to be followed for neighbor discovery. The neighbor discovery process is initiated with carrier sensing mechanism. The carrier sense mechanism is adapted for sensing the carrier and once the carrier sensed is free the discovery process is initiated.

\subsection{Neighbor Discovery Process}

The node/gateway in search of neighbors (sender node) initiates the process by broadcasting a Solicit) message. The nodes in the range receive the Solicit() messages and upon successful decoding, acknowledgment (ACK) for the receipt of Solicit() will be transmitted to the sender node. The nodes that receive the Solicit() messages compute the DOA estimate at the physical layer and find the best estimate of the signals. The information of the DOA estimation is appended to the Advertisement() message and transmitted to the sender node. The sender node receives multiple Advertisement() messages from the nodes in its vicinity (nodes in the range) and creates a neighbor table through CacheTableEntry. Fig. 2 shows the initial sequence of operations for neighbor discovery. This process is repeated by all nodes resulting in corresponding neighbors discovery. When the destination address of an incoming message is different from the receiving node's address, the receiver node determines the next-hop information by looking up the cache table and forwards the packet to the next intermediate node as shown in Fig. 3.

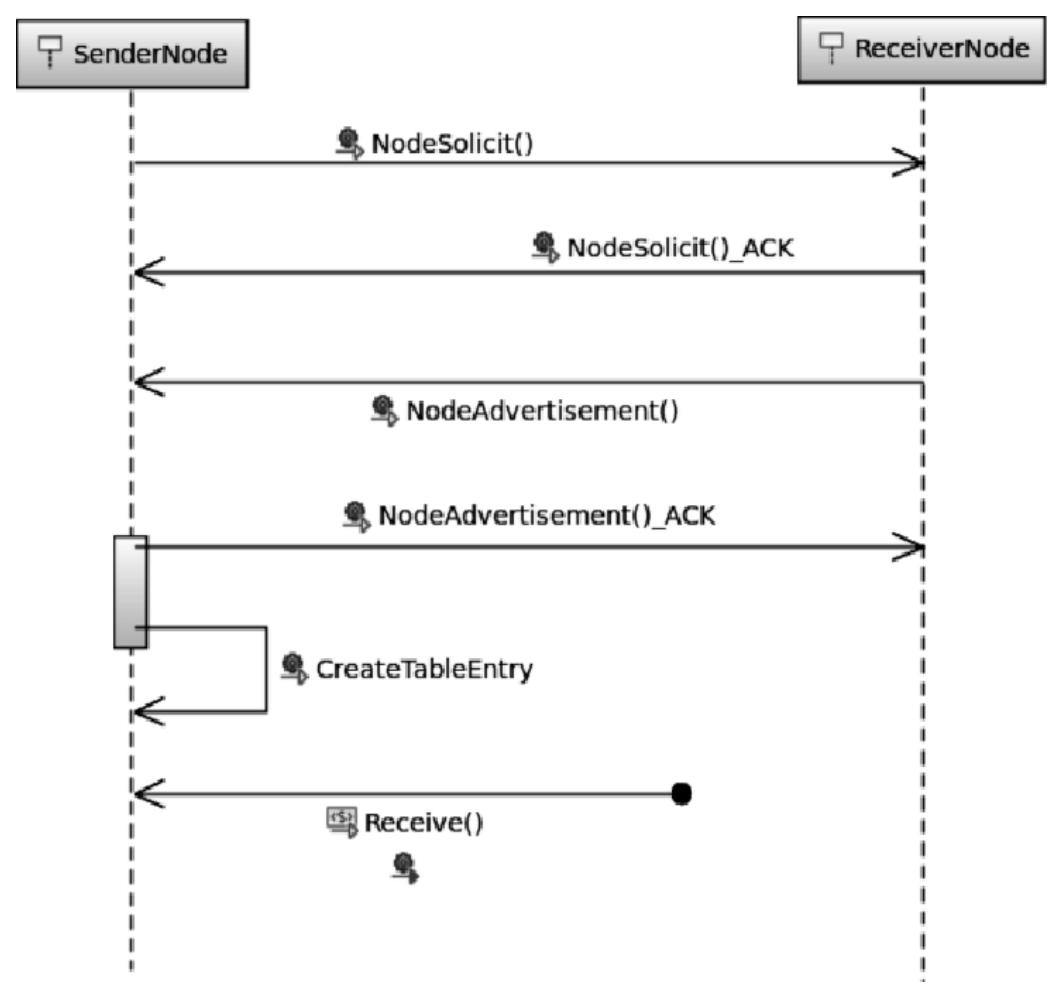

Fig. 2. Initial neighbor discovery. 


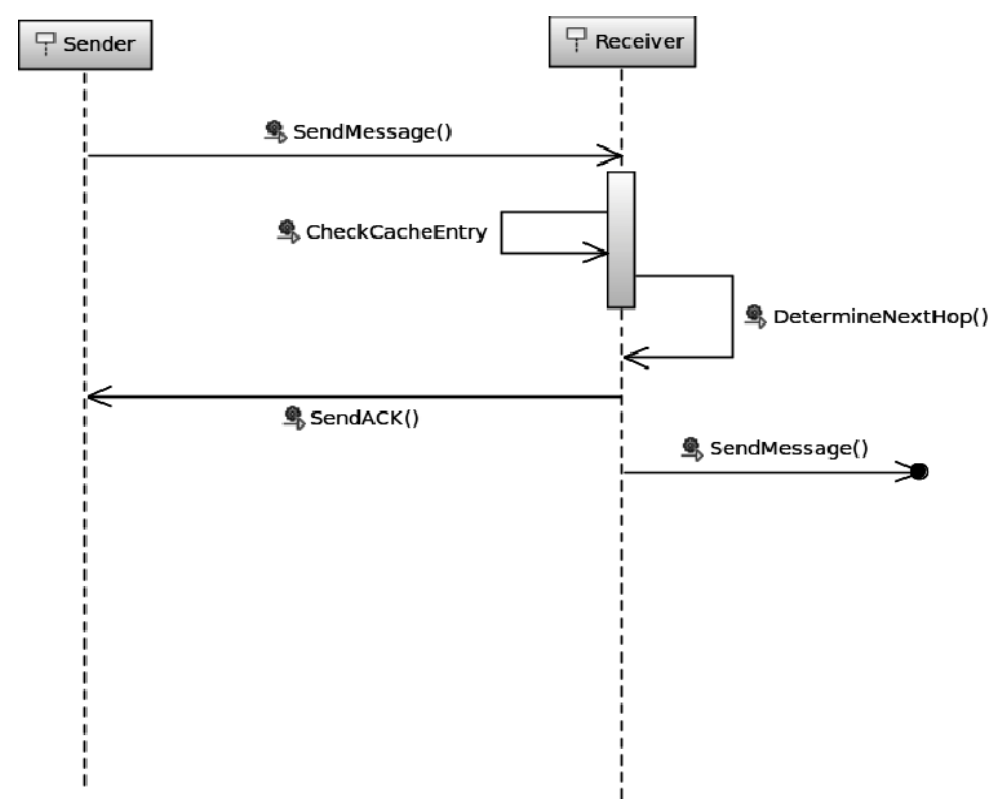

Fig. 3. Next hop determination.

\subsection{Neighbor Rediscovery Process}

The neighbor rediscovery process is initiated in two cases:

1) Failure in next hop node determination: When a node fails to determine the next-hop node in CacheTableLookup, neighbor rediscovery process is initiated. Fig. 4 shows the sequence of operations that occur when a neighbor discovery operation has to be reinitiated. This results in restarting the neighbor discovery procedure at the node locally through the exchange of Solicit() and Advertisement() messages as before. Upon successful next-hop determination, the node will forward the packet to the appropriate next-hop neighbor.

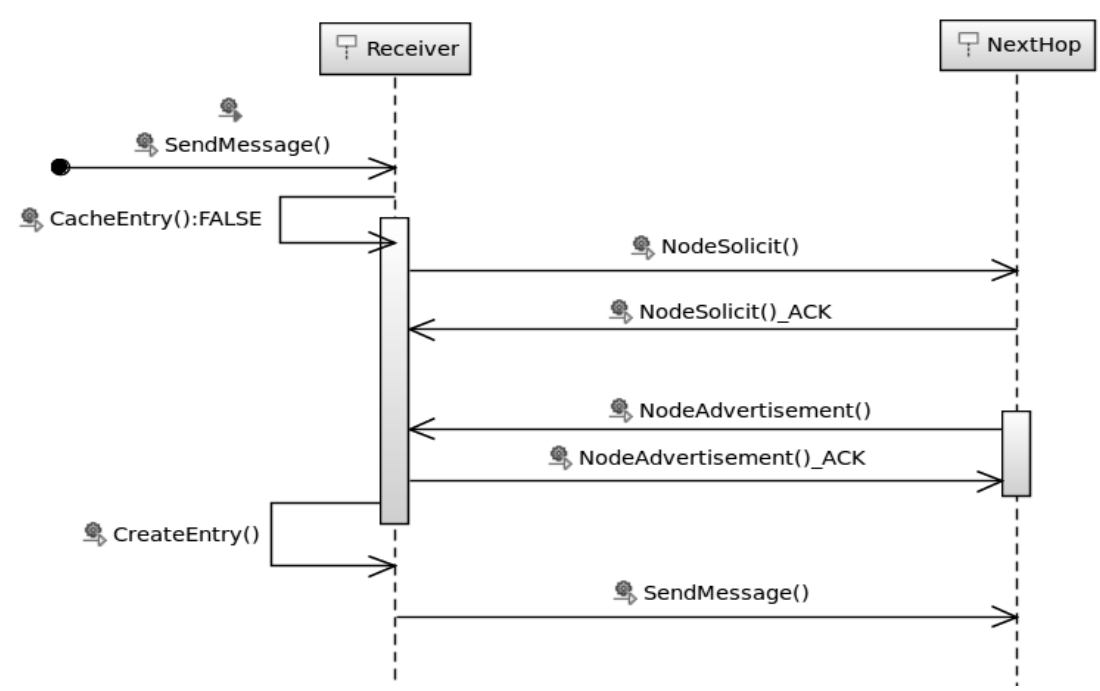

Fig. 4. CacheTableLookUp and neighbor rediscovery.

2) Unreachability detection: This process is initiated when the sender node fails to receive the acknowledgment packets before the expiry of timer. The failure to receive the acknowledgment can be due to the link failure or node failure and hence the rediscovery process has to be initiated. Fig. 5 shows a scenario where additional operations are to be performed if a node is unreachable. The sending node 
looks up at the available CacheTableLookup, forwards the packet to the nexthop address and waits for the receipt of an acknowledgment. If an ACK is not received before the Timeout at the sender node, reachability detection messages are transmitted to determine if the destination is reachable. If the destination is unreachable (no response is received) then the corresponding cache entry for that node is deleted from the CacheTableEntry and the neighbor discovery procedures are started to aid in next-hop determination.

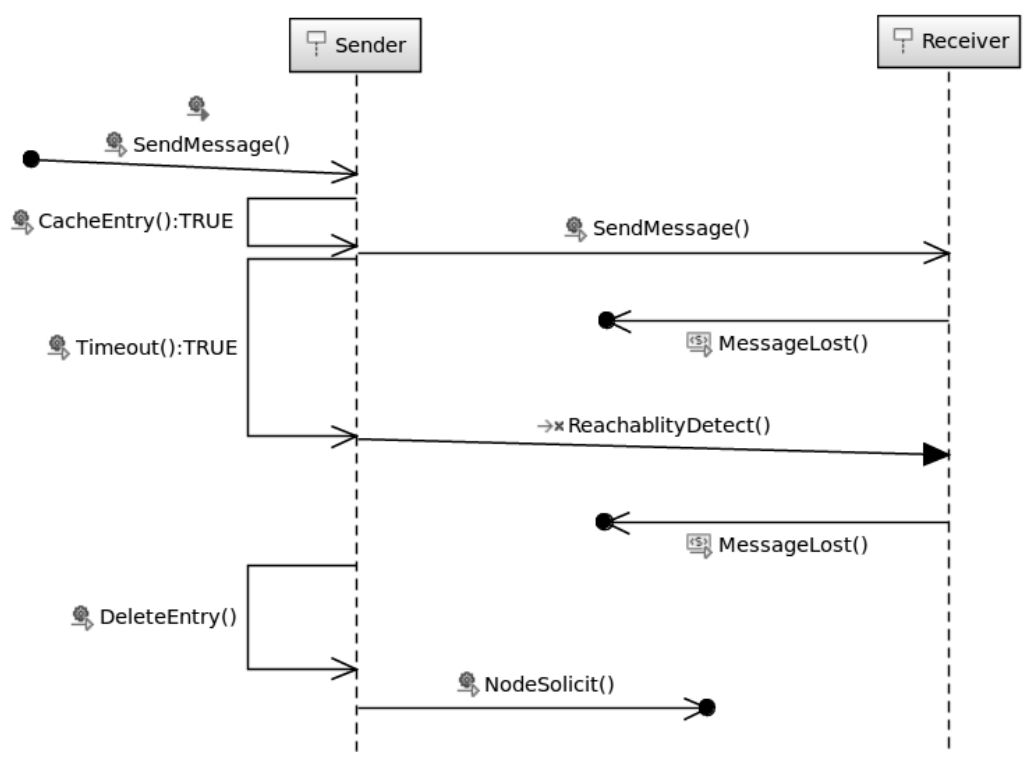

Fig. 5. Unreachability detection.

\section{Simulation Results and Discussion}

The performance of the neighbor discovery protocol is evaluated using the simulation. The simulations were run with NS3 for underwater networks. The scenario considered for simulation consists of sensor nodes and mobile nodes randomly deployed with the grid boundaries of $500 \mathrm{~m}$ by $500 \mathrm{~m}$ with a depth of $1000 \mathrm{~m}$. The mobility model chosen was the Random Waypoint mobility model with a vertical speed of 0.2 $0.4 \mathrm{~m} / \mathrm{s}$, the movement duration of 20 seconds and pause time of 5 seconds.

The physical layer also models SINR (uses pre-determined FH-FSK with ambient noise) and PER (Packet Error Rate Model) (uses FH-FSK, rate 1/2 convolutional code). The MAC layer uses the carrier sense mechanism. The simulation utilizes SeaGlider Model and Remus Model for Autonomous Underwater Vehicles (AUVs). The framework is designed to simulate AUVs behaviour. Further, the simulations were run for duration of 1000 seconds before the selection of a new random topology. We evaluate our scheme in terms of neighbor discovery and throughput of the network under varying channel conditions.

\subsection{Neighbor Discovery}

Fig. 6 presents the results for neighbor discovery for different sectoral antenna sizes of 60,90 , and 120 degrees. It can be observed that more than $98 \%$ of nodes are discovered in case of 90 and 120 degree sectors for transmission of about 10 messages as compared to $80 \%$ of nodes discovered in the sector of size 60 degree. It is due to the fact that the transmission ranges are longer for smaller sector sizes and vice-versa; and any adverse effects in channel conditions will impact smaller sectoral antenna size more as compared to larger sectoral antenna sizes.

Also, Fig. 7 shows the comparison of average number of nodes discovered vs. actual number of nodes. The comparison is made for five different cases, (1) no fading, (2) End of Cycle Packet method (ECP) [8] at 
maximal power, (3) DOA-NDP at maximal power, (4) ECP at minimal power transmissions, and (5) DOA-NDP at minimal power transmissions. It can be observed that in the first case, all nodes are discovered as none of advertisement/solicit messages are lost due to collision because of ideal channel condition. The performance of case 2 depicts similar performance in the case of maximum power transmissions, whereas the use of DOA-NDP in low power scenarios results in twice as many nodes discovered in comparison to ECP. This is due to the fact that DOA-NDP utilizes sectoral antennas and also the number of messages transmitted to discover the nodes are minimal which consequently reduces the transmission power.

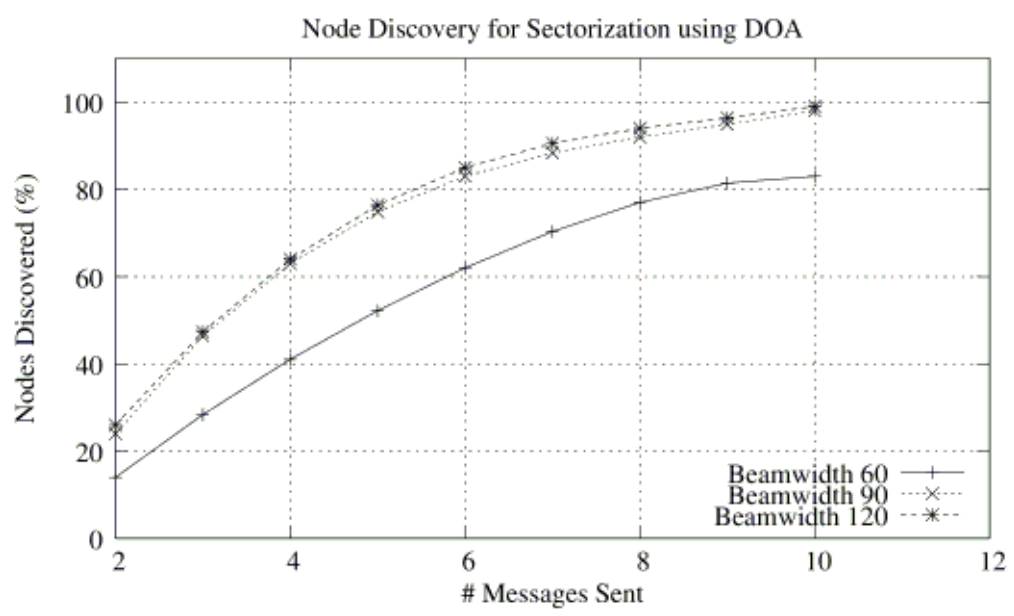

Fig. 6. Neighbor discovery using DOA-NDP.

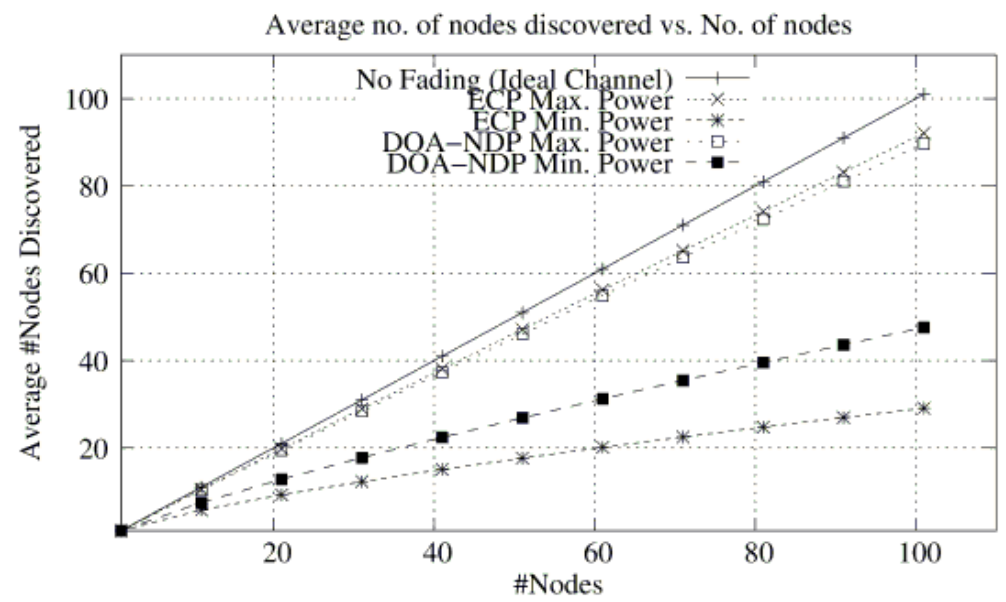

Fig. 7. Average number of nodes discovered.

\subsection{Network Throughput}

Fig. 8 shows the performance comparison for three different propagation models used with underwater acoustic networks, the Ideal channel model, Thorp propagation model and Bellhop models respectively. The throughput is higher for the ideal case as compared to the other two propogation model since no messages are lost due to ideal channel conditions. The propagation models used will involve both surface and bottom interactions, for varying environmental conditions. Each of the three propagation models provide a power delay profile (PDP) and pathloss information which is obtained using a tapped delay line model for the acoustic channel. Each delay tap has a delay and amplitude parameter depending on the time of arrival (relative to the first tap arrival time) and amplitude. The results show that thorp and bellhop models result in comparable pathloss in comparison to the ideal channel model. 


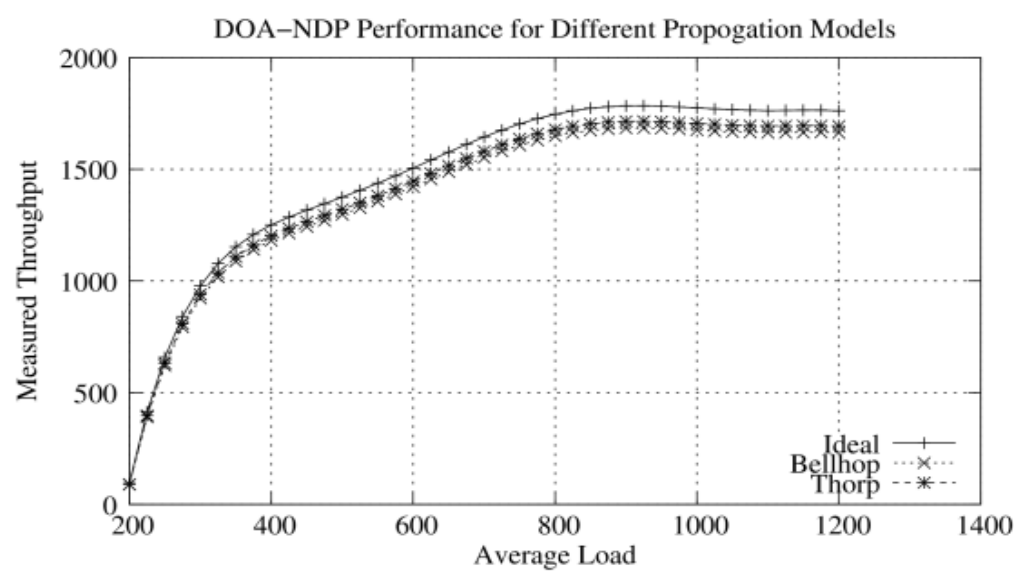

Fig. 8. Through put.

\section{Conclusion}

The paper presented the implementation design architecture of novel neighbor discovery protocol which utilizes DOA feedback. The design discusses the development of two main classes namely NeighborDiscoveryProtocol and NeighborDiscoveryCache. The protocol operations are also presented describing the functionality of the protocol including discovery procedures, rediscovery procedures (response to failure events and unreachability detection). The NDP module developed for NS3 is used to simulate the process of neighbor discovery in both static and mobile node scenarios. In both scenarios, the node deployments are randomly generated and the network performance results averaged over multiple simulation runs are presented. The results demonstrate a significant performance improvement of DOA-NDP over existing mechanisms. Further the impact of varying sector sizes on the percentage of neighbors discovered is also discussed. An energy framework for use with underwater acoustic nodes (UAN) nodes is currently under development and we are exploring the use of additional DOA algorithms and evaluating their performance in comparison to existing schemes. As a future work we plan to develop the routing scheme for underwater acoustic networks using the DOA estimation and compare with the existing routing scheme.

\section{References}

[1] Akyildiz, I. F., Pompili, D., \& Melodia, T. (2005). Underwater acoustic sensor networks: Research challenges. Ad Hoc Networks, 3(3), 257-279.

[2] Manjula, R. B., \& Manvi, S. (2011). Issues in underwater acoustic sensor networks. Journal of Computer and Electrical Engineering, 3(1), 101-111.

[3] Dimitris, V. K. I., Manolakis, G., \& Kogon, S. M. (2005). Statistical and Adaptive Signal Processing: Spectral Estimation, Signal Modeling, Adaptive Filtering and Array Processing. Artech House Print on Demand.

[4] Ramanathan, R., et al. (2005). Ad hoc networking with directional antennas: a complete system solution. IEEE Journal on Selected Areas in Communications, 23(3), 496-506.

[5] Du, J., Kranakis, E., Ponce, O. M., \& Rajsbaum, S. (2012). Neighbor discovery in a sensor network with directional antennae. Algorithms for Sensor Systems, 57-71.

[6] Jakllari, G., Luo, W., Krishnamurthy, S. V. (2007). An integrated neighbor discovery and mac protocol for ad hoc networks using directional antennas. IEEE Tran. on Wireless Communications, 6(3), 1114-1024.

[7] Pillai, U. Array Signal Processing (1st ed). New York: Springer-Verlag.

[8] Patil, A., \& Stojanovic, M. (2013). A node discovery protocol for ad hoc underwater acoustic networks. Wireless Communications and Mobile Computing, 13(3), 277-295.

[9] Proakis, M. J., \& Rice, J. (1998). Design of a communication network for shallow water acoustic modems. 


\section{IEEE International Proceedings Oceans Community Conference.}

[10] Othman, A.-K., et al (2006). Node discovery protocol and localization for distributed underwater acoustic networks. Proceedings of Int. Conf. on Int. and Web Appl. and Services/Advanced (pp. 93-93).

[11] Zhang, Y. C. (March 2010). Wormhole - Resilient secure neighbor discovery in underwater acoustic networks. Proceedings of IEEE INFOCOM (pp. 1-9).

[12] Young, B. K., Vinaychandra, S., \& Nitin, H. V. (2000). Medium access control protocols using directional antennas in Ad Hoc networks. Proceedings of IEEE Infocom.

[13] Cornejo, A., Viqar, S., \& Welch, J. L. (2014). Reliable neighbor discovery for mobile ad hoc networks. Ad Hoc Networks, 12, 259-277.

[14] Liu, D. (2009). Protecting neighbor discovery against node compromises in sensor networks. Proceedings of 29th IEEE International Conference on Distributed Computing Systems (pp. 579-588).

[15] Stoleru, R., Wu, H., \& Chenji, H. (2011). Secure neighbor discovery in mobile ad hoc networks. Proceedings of IEEE 8th International Conference on Mobile Ad Hoc and Sensor Systems (pp. 35-42).

[16] Vasudevan, S., et al (2005). On neighbor discovery in wireless networks with directional antennas. Proceedings of Annual Joint Conf. of Computer and Communications Societies: Vol 4 (pp. 2502-2512).

[17] Alonso, G., et al. (2003). Probabilistic protocols for node discovery in ad-hoc, single broadcast channel networks. Proceedings of Int. Parallel and Distributed Processing Symposium (pp. 104-115).

[18] McGlynn, M. J., et al (2001). Birthday protocols for low energy deployment and flexible neighbor discovery in ad hoc wireless networks. Proceedings of Sym. on Mob. Ad Hoc Net. \& Comp. (pp. 137-145).

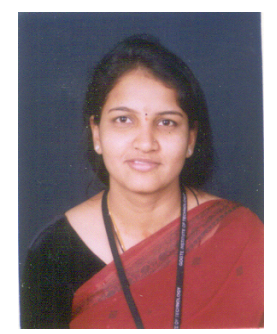

Manjula R. Bharamagoudra has completed her B.E degree in electronics and communication engineering and M.Tech degree in digital communication from Visvesvaraya Technological University, Belgaum, India and presently pursuing research in the area of underwater acoustic sensor networks. Her areas of interest include wireless communication, underwater acoustic sensor networks.

Currently she is working as a faculty member in Reva Institute of Technology and Management, REVA University, Bengaluru, India. She has around 10 national/international conference publications to her credit and few papers in national and international journals, and 2 publications of books/book-chapters. She is a member of IEEE, and ISTE (India).

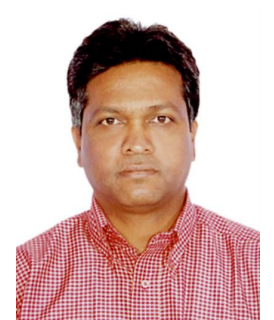

Sunilkumar S. Manvi received B.E. degree from Karnataka University, M.E. degree in electronics from University of Visweshwariah, College of Engineering, Bangalore, and Ph.D. degree in electrical communication engineering, from Indian Institute of Science, Bangalore, India. His research interests are in the area of agent based applications in multimedia communications, grid computing, ad-hoc networks, e-commerce, sensor networks and mobile computing.

He is currently working as a professor and principal of REVA Institute of Technology and Management, REVA University, Bengaluru, India. He has published 140 papers at national and international conferences, 80 papers in national and international journals, and 15 publications of books/book-chapters. He has participated in Supreme Engineers Award, Dec. 2012, ERIL India Ltd., and he has been listed in Marqui's Whos Who in the World. He was awarded the Vidya Ratan Award - 2013 by the Economic for Health and Educational Growth, New Delhi.

He is a fellow of IETE (FIETE, India), IE (FIE, India) and a member of ISTE (MISTE, India), a senior member of IEEE (SMIEEE, USA). He has executed several research projects sponsored by VTU and AICTE worth Rs. 50.00 lakhs. 